\title{
Cysteine-Rich Protein 61 (CCN1) Mediates Replicative Senescence-Associated Aberrant Collagen Homeostasis in Human Skin Fibroblasts
}

\author{
Taihao Quan," Zhaoping Qin, John J. Voorhees, and Gary J. Fisher* \\ Department of Dermatology, University of Michigan Medical School, Ann Arbor, Michigan
}

\begin{abstract}
Dermal fibroblasts produce a collagen-rich extracellular matrix, which confers mechanical strength and resiliency to human skin. During aging, collagen production is reduced and collagen fragmentation is increased, which is initiated by matrix metalloproteinase-1 (MMP-1). This aberrant collagen homeostasis results in net collagen deficiency, which impairs the structural integrity and function of skin. Cysteinerich protein 61 (CCN1), a member of the CCN family, negatively regulates collagen homeostasis, in primary human skin dermal fibroblasts. As replicative senescence is a form of cellular aging, we have utilized replicative senescent dermal fibroblasts to further investigate the connection between elevated CCN1 and aberrant collagen homeostasis. CCN1 mRNA and protein levels were significantly elevated in replicative senescent dermal fibroblasts. Replicative senescent dermal fibroblasts also expressed significantly reduced levels of type I procollagen and increased levels of MMP-1. Knockdown of elevated CCN1 in senescent dermal fibroblasts partially normalized both type I procollagen and MMP-1 expression. These data further support a key role of CCN1 in regulation of collagen homeostasis. Elevated expression of CCN1 substantially increased collagen lattice contraction and fragmentation caused by replicative senescent dermal fibroblasts. Atomic force microscopy (AFM) further revealed collagen fibril fragmentation and disorganization were largely prevented by knockdown of CCN1 in replicative senescent dermal fibroblasts, suggesting CCN1 mediates MMP-1-induced alterations of collagen fibrils by replicative senescent dermal fibroblasts. Given the ability of $\mathrm{CCN} 1$ to regulate both production and degradation of type I collagen, it is likely that elevated-CCN1 functions as an important mediator of collagen loss, which is observed in aged human skin. J. Cell. Biochem. 113: 3011-3018, 2012. (c) 2012 Wiley Periodicals, Inc.
\end{abstract}

\section{KEY WORDS: CCN1; COLLAGEN; MMP-1; SENESCENT CELLS; AGED HUMAN SKIN}

CN1 belongs to a group of proteins known as CCN family, which at present consists of six distinct members, CCN1-6 [Lau and Lam, 1999; Perbal, 2004; Leask and Abraham, 2006]. Members of the CCN family exhibit diverse cellular functions such as regulation of cell proliferation, chemotaxis, apoptosis, adhesion, motility, ion transport, and extracellular matrix (ECM) production [Lau and Lam, 1999; Brigstock, 2003; Perbal, 2004; Chen and Lau, 2009]. Like other members of CCN family, CCN1 is a secreted, ECMassociated matricellular protein. CCN1 protein has been reported to regulate cell adhesion, migration, cell-matrix interactions, and synthesis of ECM proteins in variety of cells in culture [Kireeva et al., 1996; Chen et al., 2001; Mo et al., 2002].

Type I collagen is the most abundant component of the dermal ECM in human skin. The collagenous ECM comprises the bulk of skin and is critical for structure and function. Reduced collagen production and increased fragmentation of collagen fibrils are prominent features of aged human skin. These deleterious alterations of collagen homeostasis largely account for thinning

Abbreviations: CCN1, cysteine-rich protein 61; CCN family (cysteine-rich protein 61, connective tissue growth factor, nephroblastoma overexpressed); Col-1, type I collagen; MMP-1, matrix metalloproteinase-1; ECM, extracellular matrix; PCR, polymerase chain reaction.

Conflicts of interest: None.

Additional supporting information may be found in the online version of this article.

Grant sponsor: National Institute of Health R01ES014697 R01ES014697-30S1; Grant sponsor: Dermatology Foundation Research Grant.

*Correspondence to: Taihao Quan, or Dr. Gary J. Fisher, Department of Dermatology, University of Michigan Medical School, 1301 Catherine, Medical Science I, Room 6447, Ann Arbor, MI 48109-5609. E-mail: thquan@umich.edu; gjfisher@umich.edu

Manuscript Received: 18 March 2012; Manuscript Accepted: 26 April 2012

Accepted manuscript online in Wiley Online Library (wileyonlinelibrary.com): 4 May 2012

DOI 10.1002/jcb.24179 • @ 2012 Wiley Periodicals, Inc. 
and increased fragility of skin in elderly individuals [Fisher et al., 2002; Varani et al., 2006; Fisher et al., 2009]. Little is known regarding the underlying mechanisms of age-related aberrant collagen homeostasis in human skin [Fisher et al., 2008].

We have previously reported that $\mathrm{CCN} 1$ is elevated in chronologically aged human skin, and increased levels of CCN1 negatively regulate collagen homeostasis in human dermal fibroblasts [Quan et al., 2006]. Fibroblasts are the major collagen-producing cell type in human skin. Their response to elevated CCN1 is down-regulation of type I collagen production and up-regulation of matrix metalloproteinase-1 (MMP-1), which initiates collagen degradation [Quan et al., 2006, 2010]. These data prompted us to further explore the connection between CCN1 expression and collagen homeostasis in human skin fibroblasts. To address this question, we have employed replicative senescent dermal fibroblasts, which have been extensively studied as an in vitro aging model.

\section{MATERIALS AND METHODS}

\section{CELL CULTURE AND REPLICATIVE SENESCENT DERMAL FIBROBLASTS}

Primary human skin dermal fibroblasts were prepared from healthy adult human skin biopsy and cultured as previously described [Fisher et al., 1991; Quan et al., 2005]. Briefly, human fibroblasts were isolated by digestion skin with bacterial collagenase (Worthington Biochemical Corporation, Lakewood, NJ), and cultured in Dulbecco's modified Eagle (DMEM) medium supplemented with $10 \%$ fetal bovine serum $(\mathrm{v} / \mathrm{v})$ in a humidified incubator with a $5 \% \mathrm{CO}_{2}$ atmosphere at $37^{\circ} \mathrm{C}$. Participation of human subjects was approved by the institutional review board at the University of Michigan, and all subjects provided written informed consent before entering the study. All culture reagents; DMEM, fetal bovine serum, trypsin solution, and penicillin G (100 units/ml)/streptomycin (100 units/ml) were purchased from Invitrogen Life Technology (Carlsbad, CA). Pre-senescent control dermal fibroblasts used for this study were between passages 7 and 10. Replicative senescent dermal fibroblasts were generated by serial passage of primary cultures until the cells stopped growing and expressed the phenotype of senescent cells [Going et al., 2002; Cristofalo et al., 2004], including enlarged and flattened shape, and the positivity for senescenceassociated- $\beta$-galactosidase activity (see Results for details).

\section{THREE-DIMENSIONAL COLLAGEN LATTICE CELL CULTURES}

Pre-senescent and senescent human skin dermal fibroblasts were cultured in three-dimensional collagen lattices, as previously described [Fisher et al., 2009]. Briefly, collagen lattices were prepared by mixing appropriate volume of rat tail type I collagen (BD Biosciences) with medium cocktail [DMEM, $\mathrm{NaHCO}_{3}(44 \mathrm{mmol} / \mathrm{L})$, L-glutamine ( $4 \mathrm{mmol} / \mathrm{L})$, folic acid ( $9 \mathrm{mmol} / \mathrm{L})$, and neutralized with $1 \mathrm{~N} \mathrm{NaOH}$ to $\mathrm{pH}$ 7.2] to yield a final concentration of $2 \mathrm{mg} / \mathrm{ml}$. Fibroblasts $\left(2.5 \times 10^{5}\right)$ were suspended in $2 \mathrm{ml}$ collagen solution in $35 \mathrm{~mm}$ culture dish, and placed in an incubator at $37^{\circ} \mathrm{C}$ for $30 \mathrm{~min}$ to allow polymerization of the collagen. After polymerization, collagen lattices were detached from sides and bottom of the dish, covered with $2 \mathrm{ml}$ of media (DMEM, 10\% fetal bovine serum) and incubated for $24 \mathrm{~h}$ at $37^{\circ} \mathrm{C}, 5 \% \mathrm{CO}_{2}$. To activate secreted MMP-1, collagen lattices were washed extensively with PBS (at least three times for $30 \mathrm{~min}$ ), and then treated with Trypsin-EDTA $(100 \mathrm{ng} / \mathrm{ml}$, Invitrogen) in serum-free media for $24 \mathrm{~h}$. Conditioned media were collected, concentrated, and analyzed by 10\% SDS-PAGE. Collagen bands were visualized by staining with SimplyBlue (Invitrogen).

\section{RNA ISOLATION AND QUANTITATIVE REAL-TIME RT-PCR}

Total RNA was isolated using a commercial kit (RNeasy midikit, Qiagen, Chatsworth, CA) according to the manufacturer's protocol. Total RNA (100 ng) was reverse transcribed using Taqman Reverse Transcription kit (Applied Biosystems, Foster City, CA). Real-time PCR was performed on a 7300 Sequence Detector (Applied Biosystems) using Taqman Universal PCR Master Mix Reagents (Applied Biosystems). PCR primers and probes were purchased from Applied Biosystems. Type I procollagen, MMP-1, CCN1, and 36B4 primers and probes were described previously [Fisher et al., 2009; Quan et al., 2004, 2006, 2009]. Integrin primers were ordered from Realtimeprimers.com. Integrin $\alpha 1$ (ITGA1), forward primer, 5' - CAAACTGCAGACCCCATATC-3', reverse primer, 5' - TGACTTGGCTGATGTCAGAA-3'; Integrin $\alpha 2$ (ITGA2), forward primer, $5^{\prime}-$ ATGCAGATGGACCACACTTT-3', reverse primer, 5' ${ }^{\prime}$-AAGCATCACTGCTGAACTCC-3'; Integrin $\alpha 10$ (ITGA10), forward primer, 5'-TGATTTTCTGAAGCCAGGAG-3', reverse primer, 5'-AAAGCATTTCCCATCATCAA-3', Integrin $\alpha 11$ (ITGA11), forward primer, 5'-AAGTCACACCСАСТСССТTC-3', reverse primer: 5'-ATGTGTGAGTTGCGGTCAAT-3'. Target gene levels were normalized to the housekeeping gene 36B4, as an internal control for quantification.

\section{WESTERN BLOT ANALYSIS}

Western analysis was performed as previously described [Quan et al., 2001]. Briefly, whole cell extract was prepared from the cells using whole cell extraction buffer (25 mM HEPES, 0.3 M NaCl, $1.5 \mathrm{mM}$ $\mathrm{MgCl}_{2}, 0.2 \mathrm{mM}$ EDTA, $0.1 \%$ Triton X-100, $0.5 \mathrm{mM}$ DTT, $20 \mathrm{mM}$ $\beta$-glycerolphosphate, $0.1 \mathrm{mM} \mathrm{Na} \mathrm{VO}_{4}, 2 \mu \mathrm{g} / \mathrm{ml}$ leupeptin, and $100 \mu \mathrm{g} / \mathrm{ml}$ PMSF). Whole cell extract was prepared by centrifugation, and concentrations of proteins were measured by Bio-Rad protein assay (Bio-Rad Laboratories, Hercules, CA). Proteins were resolved on 10\% SDS-PAGE, transferred to PVDF membrane, and reacted with primary antibodies. Type I procollagen antibody was purchased from Southern Biotech (Birmingham, AL) and MMP-1 and CCN1 antibodies were purchased from Santa Cruz Biotechnology (Santa Cruz, CA). Protein bands were visualized and quantified with enhanced chemifluorescence (ECF) (Vistra ECF Western Blotting System, GE Healthcare, Piscataway, NJ) following the manufacturer's protocol. The intensities of each band were quantified by STORM PhosphorImager (Molecular Dynamics, Sunnyvale, CA) and normalized using $\beta$-actin as loading control.

\section{ENZYME-LINKED IMMUNOSORBENT ASSAY (ELISA) AND SECRETED CCN1 PROTEIN}

Secreted type I procollagen and MMP-1 were determined from fibroblast culture fluids using enzyme-linked immunosorbent assay kits (type I procollagen, Takara Mirus Bio USA, Madison, WI; human MMP-1, GE HealthCare) according to manufacturer's instructions. 
Secreted CCN1 protein was determined from fibroblasts culture fluids by western blot analysis as described above.

\section{CELLTRACKER AND $\boldsymbol{\beta}$-GAL STAINING}

Fibroblast morphology was assessed by incubation of cultures with CellTracker fluorescent dye (Molecular Probes, Eugene, OR) for $1 \mathrm{~h}$ [Fisher et al., 2009]. The cells were washed with PBS and were fixed in $2 \%$ paraformaldehyde for $30 \mathrm{~min}$. Fibroblasts were imaged by fluorescence microscopy. Senescence-associated- $\beta$-galactosidase staining was performed using kit (Senescence $\beta$-gal Staining Kit, Cell Signaling Technology Inc, Danvers, MA) according to manufacturer's instructions.

\section{TRANSFECTION AND SIRNA}

Human skin fibroblasts were transiently transfected with control siRNA (5'-UUCUCCGAACGUGUCACGU-3') and CCN1 siRNA (5'GCUGCGUUUCCCUUCUACU- $3^{\prime}$ ) as described previously [Quan et al., 2010]. siRNAs were synthesized from Qiagen (Chatsworth, CA), and transfection was performed by electroporation (Amaxa Biosystems, Koeln, Germany) according to the manufacturer's protocols. After $48 \mathrm{~h}$ of transfection, whole cell extract was prepared and protein levels were determined by western blot analysis, as described above.

\section{ATOMIC FORCE MICROSCOPY (AFM) IMAGING}

Three-dimensional collagen lattices were washed with PBS and mounted on microscope glass slides $(25 \times 75 \times 1.0 \mathrm{~mm}$, Fisher Scientific, Pittsburgh, PA), and allowed to air dry for at least $48 \mathrm{~h}$ before AFM image analysis. AFM images were obtained using a Dimension Icon AFM system (Bruker-AXS, Santa Barbara, CA), as previously described [Quan et al., 2011ab]. Briefly, the scan positions of the collagen lattices were determined by light optical image. AFM images were obtained with ScanAsyst mode in air using a silicon etched cantilever (NSC15/AIBS, MikroMasch, San Jose, CA) with a full tip cone angle $\sim 40^{\circ}$ and the tip radius of curvature $\sim 10 \mathrm{~nm}$. AFM images were acquired at a scan rate of $0.977 \mathrm{~Hz}$, $512 \times 512$ pixel resolutions. AFM imaging was conducted at the Electron Microbeam Analysis Laboratory (EMAL), University of Michigan College of Engineering, and analyzed using Nanoscope Analysis software (Nanoscope Analysis v120R1sr3, Bruker-AXS, Santa Barbara, CA).

\section{STATISTICAL ANALYSIS}

Data are expressed as mean \pm SEM. Student's $t$-test was used to evaluate the statistical differences between the groups. All $P$-values are two-tailed, and less than 0.05 was considered statistically significant.

\section{RESULTS}

\section{ESTABLISHMENT OF REPLICATIVE SENESCENT DERMAL FIBROBLASTS CULTURES}

After multiple serial passages, primary human skin dermal fibroblasts eventually stop growing and reach a state of replicative senescence [Going et al., 2002; Cristofalo et al., 2004]. We found that primary human dermal fibroblasts undergo replicative senescence after 6 months of weekly passage, which is approximately 100 population doublings. Senescent cells display a well-defined morphological and molecular signature characterized by enlarged and flattened shape and expression of senescence-associated $\beta$-galactosidase activity, a biomarker of cellular senescence [Dimri et al., 1995]. A red fluorescent dye (CellTracker), which is taken up into the cell cytoplasm was used to assess the morphology of dermal fibroblasts [Fisher et al., 2009]. Figure 1A shows that replicative senescent dermal fibroblasts display an enlarged and flattened shape. Quantitative morphometric analysis revealed that surface area of senescent fibroblasts was increased threefold, compared to pre-senescent dermal fibroblasts. Figure 1B shows that senescent dermal fibroblasts displayed substantial positive staining of senescence-associated $\beta$-galactosidase, while pre-senescent dermal fibroblasts were negative.

\section{CCN1 IS INCREASED IN REPLICATIVE SENESCENT DERMAL FIBROBLASTS}

CCN1 is elevated in aged dermal fibroblasts in vivo, and overexpression of CCN1 negatively regulates collagen homeostasis in

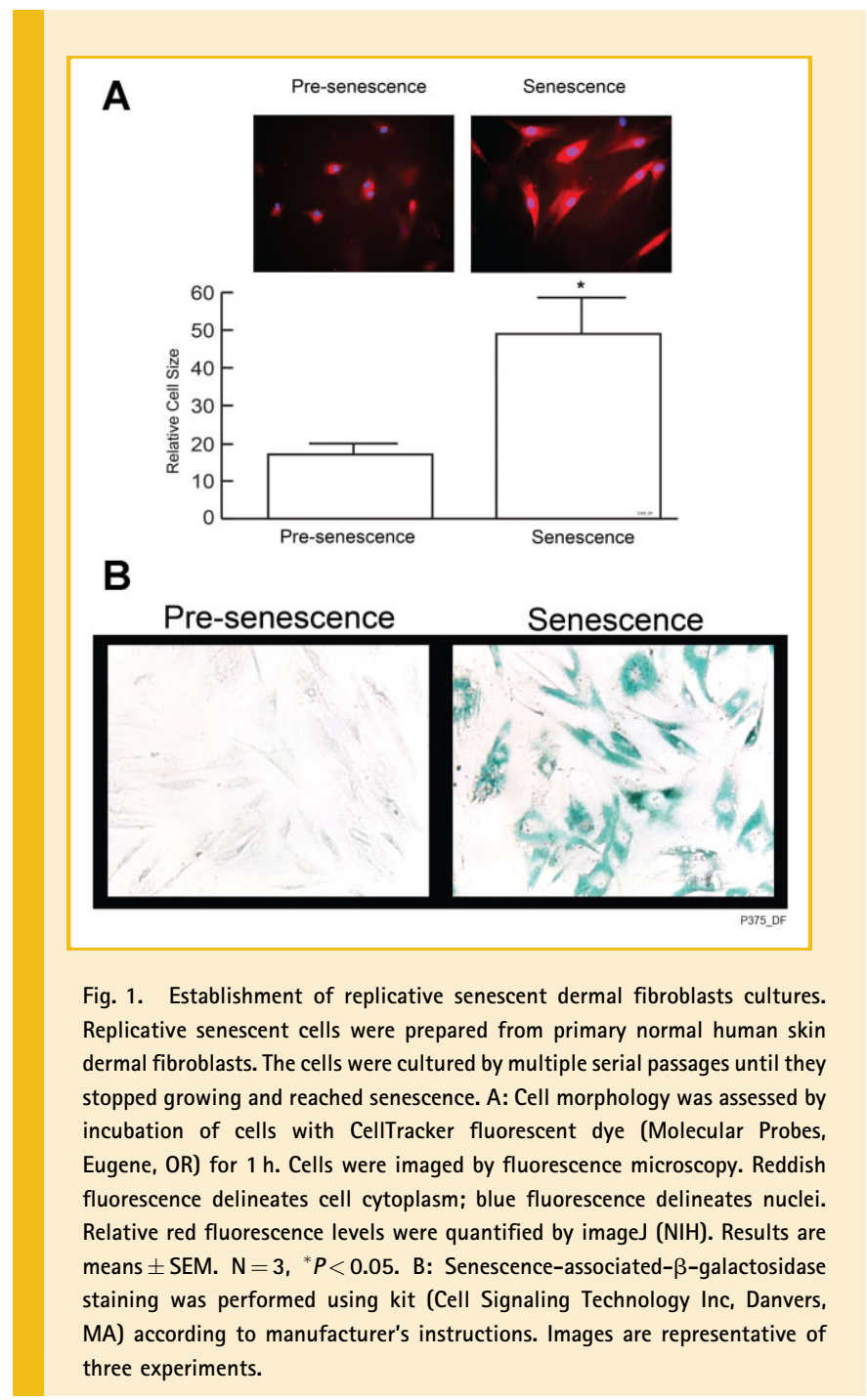


human skin dermal fibroblasts [Quan et al., 2006]. We found that CCN1 expression was significantly elevated in senescent dermal fibroblasts. Both CCN1 mRNA (Fig. 2A), and intracellular (Fig. 2B) protein were increased threefold, and extracellular secreted CCN1 protein was elevated fourfold (Fig. 2C) in senescent dermal fibroblasts, compared to pre-senescent dermal fibroblasts.

\section{CCN1 REGULATES INCREASED MMP-1 EXPRESSION IN REPLICATIVE SENESCENT DERMAL FIBROBLASTS}

MMP-1 is the major enzyme that initiates degradation of collagen fibrils, in human skin [Fisher et al., 1997; Brennan et al., 2003; Fligiel et al., 2003]. We found that MMP-1 expression was significantly elevated in senescent dermal fibroblasts. Both MMP-
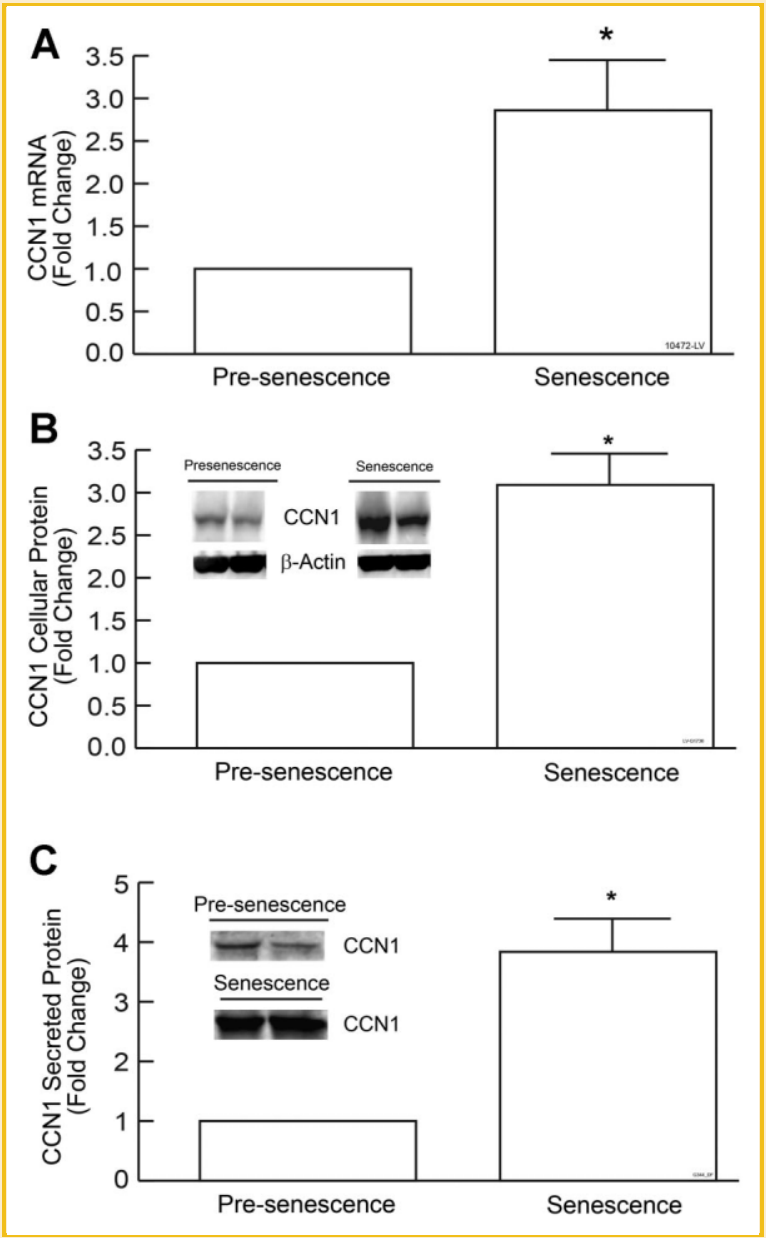

Fig. 2. $\quad$ CCN1 is increased in replicative senescent dermal fibroblasts. A: CCN1 mRNA levels were quantified by real-time RT-PCR and were normalized to mRNA for 36B4, a ribosomal protein used as an internal control for quantitation. Data are expressed as mean $\pm \mathrm{SEM}, \mathrm{N}=3,{ }^{*} P<0.05$. B: Intracellular CCN1 protein levels were determined by western analysis and the intensities were quantified and normalized using $\beta$-actin as loading control. Insets show representative western blots. Data are expressed as mean $\pm S E M, N=3$, ${ }^{*} P<0.05$. C: Secreted CCN1 protein levels from culture media were determined by western blot analysis. Same amount of proteins were resolved on $10 \%$ SDS-Page. The intensities were quantified and normalized by cell numbers. Data are expressed as mean $\pm \mathrm{SEM}, \mathrm{N}=3,{ }^{*} P<0.05$.
1 mRNA (Fig. 3A), and intracellular (Fig. 3B) protein were increased greater than threefold, and extracellular secreted MMP-1 protein was elevated greater than fourfold (Fig. 3C) in senescent dermal fibroblasts, compared to pre-senescent dermal fibroblasts. We next investigated the connection between elevated expression of $\mathrm{CCN} 1$

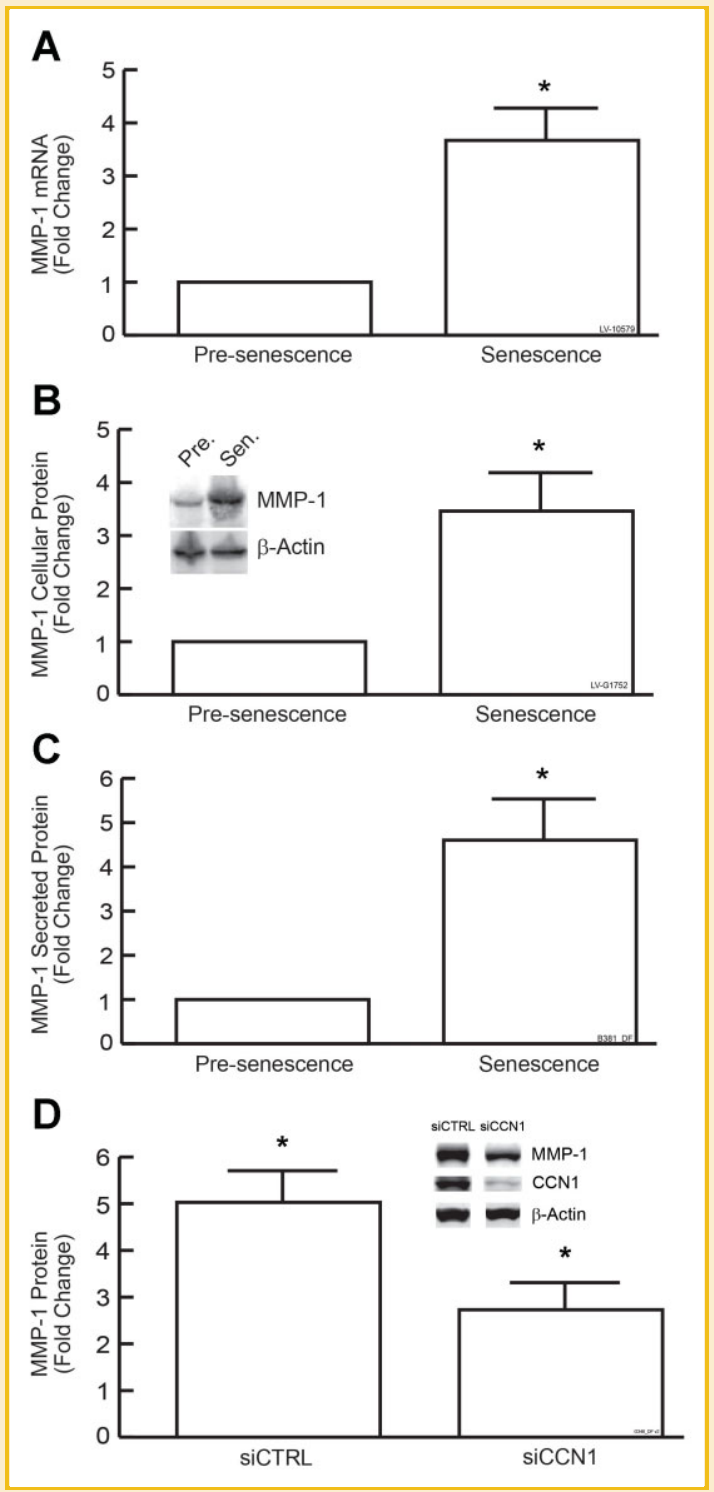

Fig. 3. CCN1 regulates increased MMP-1 expression in replicative senescent dermal fibroblasts. A: MMP-1 mRNA levels were quantified by real-time RTPCR and were normalized to mRNA for 36B4, a ribosomal protein used as an internal control for quantitation. Data are expressed as mean $\pm S E M, N=3$, ${ }^{*} P<0.05$. B: Intracellular MMP-1 protein levels were determined by western blot analysis and the intensities were quantified and normalized using $\beta$-actin as loading control. Insets show representative western blots. Data are expressed as mean $\pm \mathrm{SEM}, \mathrm{N}=3,{ }^{*} P<0.05$. C: Secreted MMP-1 protein levels from culture media were determined by ELISA. Data are expressed as mean $\pm \mathrm{SEM}$, $\mathrm{N}=3,{ }^{*} P<0.05$. D: Senescent dermal fibroblasts were transfected with control siRNA or CCN1 siRNA. MMP-1 and CCN1 protein levels were determined 2 days after transfection by western blot analysis. Insets show representative western blots. Data are means $\pm \mathrm{SEM}, \mathrm{N}=3,{ }^{*} \mathrm{P}<0.05$. 
and MMP-1 in senescent dermal fibroblasts, using siRNA-mediated knockdown. Treatment of senescence dermal fibroblasts with CCN1 siRNA reduced CCN1 protein levels greater than 90\%. Knockdown of CCN1 in senescent dermal fibroblasts reduced elevated MMP-1 protein levels 50\% (Fig. 3D). These data support the concept that elevated CCN1 is an important mediator of elevated MMP-1 in replicative senescent dermal fibroblasts.

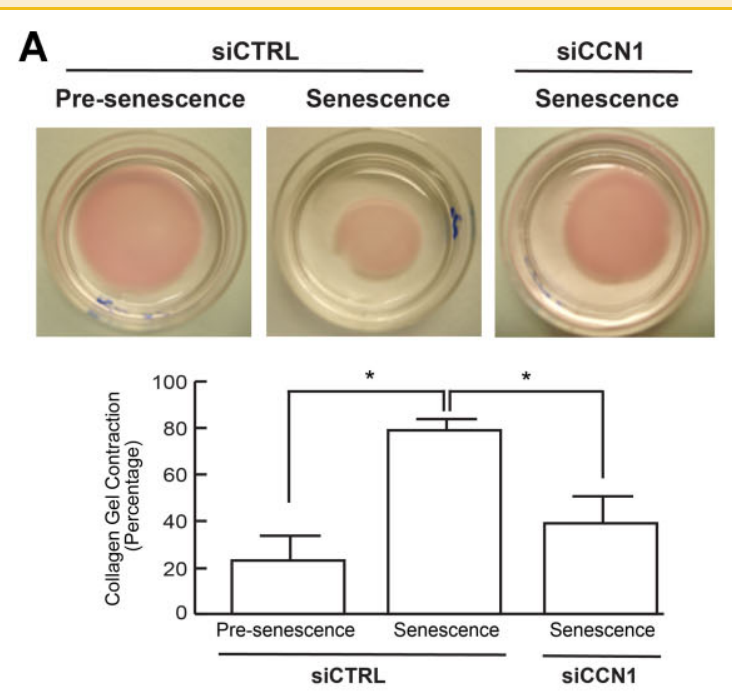

B

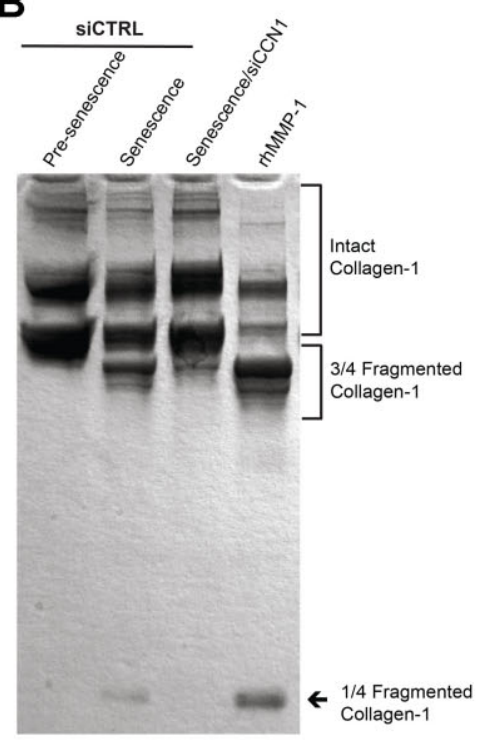

C

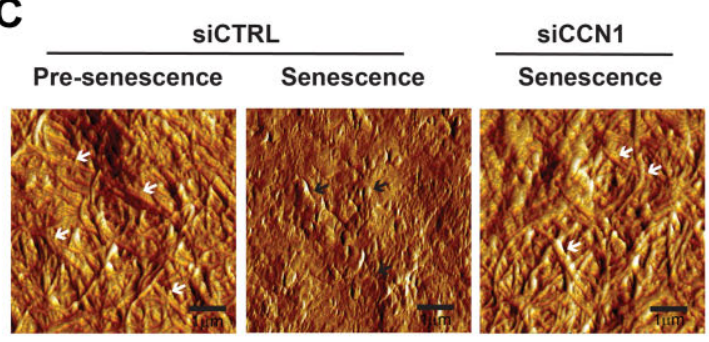

\section{CCN1 MODULATES COLLAGEN GEL, CONTRACTION AND ALTERATIONS INDUCED BY RELICATIVE SENSCENCE DERMAL FIBROBLASTS}

Cleavage of collagen fibrils by MMP-1 promotes contraction of dermal equivalent cultures, which are composed of dermal fibroblasts embedded in three dimensional collagen lattices. Figure 4A demonstrates that replicative senescent fibroblasts contracted three-dimensional collagen lattices significantly greater than pre-senescent fibroblasts. This increased lattice contraction was inhibited $72 \%$ by knockdown of CCN1 (Fig. 4A) and 78\% by MMP inhibitor MMI270 (33 nM), suggesting that CCN1-induced MMP-1 plays a major role in senescence-mediated collagen gel contraction. MMP-1 cleaves collagen fibrils at a single site, resulting in one-quarter and three-quarter length fragments [Fligiel et al., 2003; Varani et al., 2008]. We therefore examined the pattern of collagen fragmentation in dermal equivalent cultures containing pre-senescent or replicative senescent fibroblasts. Cultures containing pre-senescent fibroblasts displayed nearly undetectable collagen fragments (Fig. 4B), consistent with lack of contraction of these cultures. Cultures containing senescent fibroblasts, however, displayed readily detectable collagen fragments, consistent with substantial contraction of these cultures. This accumulation of MMP-1-generated collagen fragments was substantially reduced by knockdown of CCN1 in senescent dermal fibroblasts (Fig. 4B), further supporting the role of CCN1 in upregulation of MMP-1 expression. Finally, we used atomic force microscopy (AFM) to assess the structure of collagen fibrils in dermal equivalent cultures containing pre-senescent or replicative senescent dermal fibroblasts. AFM revealed that collagen lattices containing pre-senescent fibroblasts were composed of long, intertwining, intact collagen fibrils (Fig. 4C). In contrast, collagen fibrils in lattices containing senescent fibroblasts were largely fragmented and disorganized, consistent with biochemical measurement of increased collagen fragmentation. This collagen fibril fragmentation was largely prevented by knockdown of CCN1 in replicative senescent dermal fibroblasts (Fig. 4C). Taken together, these data indicate that upregulation of CCN1 induces collagen fragmentation by MMP-1, which mediates collagen lattice disorganization and contraction by replicative senescent dermal fibroblasts.

Fig. 4. CCN1 modulates collagen gel contraction and alterations induced by replicative senescent dermal fibroblasts. Pre-senescent and replicative senescent dermal fibroblasts were transfected with control siRNA or CCN1 siRNA. One day after transfection, the cells were cultured in three-dimensional collagen lattices for $24 \mathrm{~h}$. Secreted MMP-1 was activated by Trypsin-EDTA for $24 \mathrm{~h}$ as described in Materials and Methods section. A: CCN1 modulates collagen gel contraction. Collagen gel contraction was imaged by digital camera and quantified. Data expressed as mean $\pm \mathrm{SEM}, \mathrm{N}=3,{ }^{*} P<0.05$. B: CCN1 modulates collagen fragmentations. Conditioned media were collected, concentrated, and resolved in a 10\% SDS-PAGE. Intact and fragmented collagens were visualized by staining with SimplyBlue. Activated recombinant human MMP-1 (rhMMP-1) used as a positive control. C: CCN1 modulates collagen disorganization. Nanoscale three-dimensional collagen lattices were visualized by atomic force microscopy (AFM) as described in Materials and Methods section. The white arrows indicate intact and well-organized collagen fibrils and black arrows indicate damaged and disorganized collagen fibrils. AFM images are representative of three independent experiments. 


\section{CCN1 REGULATES REDUCED TYPE I PROCOLLAGEN EXPRESSION IN REPLICATIVE SENESCENT DERMAL FIBROBLASTS}

Type I collagen fibrils are the major structural component of the dermal ECM. We found that type I procollagen expression was significantly reduced in senescent dermal fibroblasts. Both type I procollagen mRNA (Fig. 5A), and intracellular (Fig. 5B) protein were reduced greater than $80 \%$, and extracellular secreted type I procollagen protein was reduced nearly 70\% (Fig. 5C) in senescent dermal fibroblasts, compared to pre-senescent dermal fibroblasts. Importantly, knockdown of CCN1 significantly increased type I procollagen protein levels in senescent dermal fibroblasts (Fig. 5D). These data support the concept that elevated CCN1 is an important mediator of type I collagen homeostasis in replicative senescent human dermal fibroblasts.

\section{DISCUSSION}

Type I collagen fibrils form the framework of the ECM in the dermal compartment of skin. The collagenous ECM is critical for normal skin structure and function. Fibroblasts within the dermis are primarily responsible for maintaining ECM homeostasis by controlling the production and turnover of type I collagen fibrils. During the passage of time, collagen fibrils undergo progressive alterations characterized by reduced organization, thinning, and increased fragmentation [Quan et al., 2006; Varani et al., 2006; Fisher et al., 2009; Quan et al., 2010]. These alterations are associated with decreased collagen synthesis and increased expression of collagen-degrading MMPs, by dermal fibroblasts. Deleterious alterations of skin connective tissue collagen impair skin structural integrity, resulting in increased skin fragility, which impairs wound healing [Eaglstein, 1986; Holt et al., 1992; RaineFenning et al., 2003]. Age-dependent alterations in the dermal ECM also create a microenvironment that facilitates formation and growth of skin cancer [Weinstock, 1994; Khorramizadeh et al., 1999; Campisi, 2003].

While impaired dermal fibroblast function is largely responsible for aberrant collagen homeostasis in aged skin, the underlying mechanisms are not well understood. We find that CCN1 is a negative regulator of collagen homeostasis in dermal fibroblasts, and that CCN1 is substantially elevated in aged human skin [Quan et al., 2006, 2011a]. This observation led us to explore the relationship between age-related elevation of CCN1 and aberrant collagen homeostasis in human skin fibroblasts. To address this question, we employed a replicative senescent dermal fibroblast model. Aging of cultured cells in vitro is often used as a model of cellular aging [Dimri et al., 1995; Going et al., 2002; Cristofalo et al., 2004]. We find that replicative senescent dermal fibroblasts resemble fibroblasts in aged human skin in vivo, with respect to reduced expression of type I procollagen and elevated MMP-1, as well as elevated CCN1. Importantly, we find that aberrant collagen homeostasis in replicative senescent dermal fibroblasts is partially normalized by knockdown of elevated CCN1, indicating elevated $\mathrm{CCN} 1$ functions as a mediator of cellular senescence-associated alterations in collagen homeostasis.

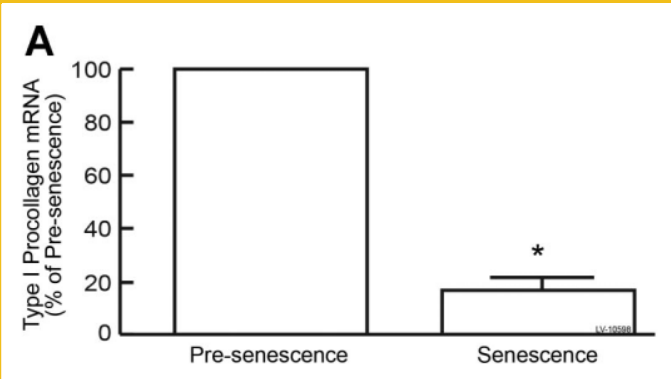

B
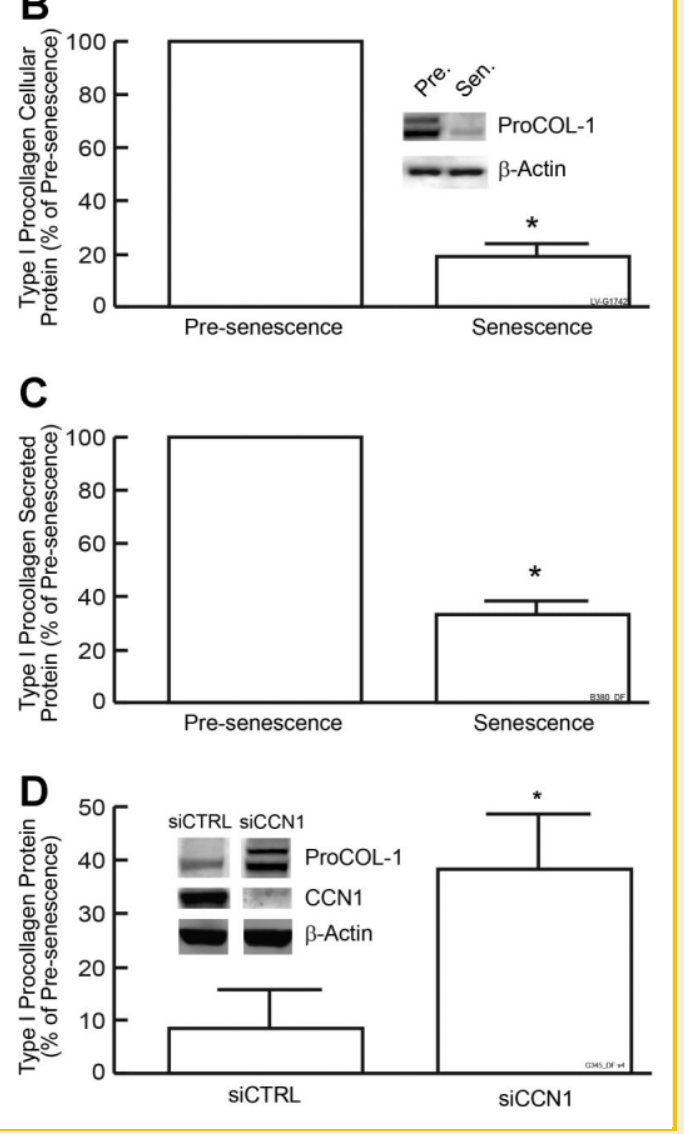

Fig. 5. Type I procollagen is reduced in replicative senescent dermal fibroblasts. A: Type I procollagen mRNA levels were quantified by real-time RT-PCR and were normalized to $\mathrm{mRNA}$ for $36 \mathrm{~B} 4$, a ribosomal protein used as an internal control for quantitation. Data are expressed as mean $\pm \mathrm{SEM}, \mathrm{N}=3,{ }^{*} P<0.05$. $\mathrm{B}$ : Intracellular type I procollagen (COL-1) protein levels were determined by western blot analysis and the intensities were quantified and normalized using $\beta$-actin as loading control. Insets show representative western blots. Data are expressed as mean $\pm \mathrm{SEM}, \mathrm{N}=3,{ }^{*} P<0.05$. C: Secreted type I procollagen protein levels from culture media were determined by ELISA. Data are expressed as mean $\pm \mathrm{SEM}, \mathrm{N}=3,{ }^{*} P<0.05$. D: Senescent dermal fibroblasts were transfected with control siRNA or CCN1 siRNA. Type I procollagen and CCN1 protein levels were determined 2 days after transfection by western blot analysis. Insets show representative western blots. Data are means \pm SEM, $N=3,{ }^{*} P<0.05$.

We have previously reported that $\mathrm{CCN} 1$ down regulates TGF- $\beta$ type II receptor, thereby impairing TGF- $\beta 1$ responsiveness in human dermal fibroblasts [Quan et al., 2006]. It is well established that TGF$\beta 1$ controls both collagen production and collagen degradation 
[Varga et al., 1987; Chen et al., 2000; White et al., 2000; Hall et al., 2003]. TGF- $\beta 1$ stimulates collagen synthesis and down-regulates MMP- 1 expression. TGF- $\beta 1$ also up-regulates plasminogen activator inhibitor-1 and tissue inhibitor of metalloproteinase, two major inhibitors of ECM-degrading enzymes. In addition we reported that elevated CCN1 induces AP-1 transcription factor [Quan et al., 2006], which directly drives MMP-1 transcription, and negatively regulates type I procollagen expression [Matrisian, 1994; Fisher and Voorhees, 1998; Fisher et al., 2000]. Therefore, it is likely that elevated CCN1 alters collagen homeostasis by impairment of TGF- $\beta$ signaling and induction of transcription factor $\mathrm{AP}-1$, in replicative senescent dermal fibroblasts.

Molecular mechanisms responsible for the elevated expression of CCN1 in replicative senescent dermal fibroblasts remain to be determined. Interestingly, replicative senescence is associated with increased oxidative stress [Campisi, 2003; Brandl et al., 2011], and we have observed that CCN1 is markedly induced by oxidative stress (unpublished data). These data raise the possibility that CCN1 is induced by oxidative stress in replicative senescent dermal fibroblasts.

CCN1 belongs to a family of secreted proteins termed matricellular proteins [Lau and Lam, 1999; Perbal, 2004; Leask and Abraham, 2006]. Fibroblasts secrete CCN1 where it interacts with the extracellular milieu, including ECM, growth factors, proteases, and cytokines. Analysis of mechanisms by which CCN1 functions indicates that it exerts cellular actions primarily through interactions with cell surface adhesion receptors, integrins [Lau and Lam, 1999; Chen and Lau, 2009]. It appears that CCN1 interacts with distinct integrins depending on the cell type and that these interactions provide functional specificity. At least seven integrins have been shown to mediate CCN1 functions in cell type specific manner [Chen and Lau, 2009]. In fibroblasts, CCN1 mediates cell adhesion and migration through $\alpha v \beta 5$ and $\alpha v \beta 3$ integrins, respectively. The exact complement of integrins that mediate CCN1 regulation of collagen homeostasis in human dermal fibroblasts remains to be determined.

Binding of fibroblasts to collagen is required for lattice contraction. This binding is mediated by specific collagen receptors, integrins $\alpha 1 \beta 1, \alpha 2 \beta 1$, and $\alpha 11 \beta 1$ [Klein et al., 1991; Tiger et al., 2001; Jokinen et al., 2004]. We find that knockown of CCN1 does not alter expression of collagen-binding integrins, in senescent dermal fibroblasts (see supplemental figure). Collagen gel contraction operates against the mechanical properties of the collagen fibrils. Intact collagen fibrils provide greater resistance than fragmented collagen fibrils to the contractive mechanical forces exerted on them by dermal fibroblasts. MMP-1-mediated collagen fragmentation promotes collagen gel contraction [Fisher et al., 2009]. Therefore, we conclude that MMP-1-catalyzed collagen fragmentation plays a major role in CCN1-mediated collagen gel contraction.

Dermal fibroblasts in aged human skin express elevated levels of CCN1 [Quan et al., 2006], and aberrant collagen homeostasis characterized by reduced type I procollagen [Quan et al., 2010] and increased MMP-1 expression [Fisher et al., 2009]. The data presented above support the concept that aberrant collagen homeostasis in aged human skin in mediated, at least in part, by elevated CCN1. Suppression of CCN1 expression may provide a novel therapeutic mechanism to improve the health of chronologically aged human skin and age-related skin diseases.

\section{ACKNOWLEDGMENTS}

We thank Diane Fiolek for graphics and administrative assistance and Suzan Rehbine for the procurement of tissue specimens. This work was supported by the National Institute of Health (grant R01ES014697 and R01ES014697-30S1 to T Quan), and Dermatology Foundation Research Grant (to T Quan).

\section{REFERENCES}

Brandl A, Meyer M, Bechmann V, Nerlich M, Angele P. 2011. 0xidative stress induces senescence in human mesenchymal stem cells. Exp Cell Res 317: 1541-1547.

Brennan M, Bhatti H, Nerusu KC, Bhagavathula N, Kang S, Fisher GJ, Varani J, Voorhees JJ. 2003. Matrix metalloproteinase-1 is the major collagenolytic enzyme responsible for collagen damage in UV-irradiated human skin. Photochem Photobiol 78:43-48.

Brigstock DR. 2003. The CCN family: A new stimulus package. J Endocrinol 178:169-175.

Campisi J. 2003. Cancer and ageing: Rival demons? Nat Rev Cancer 3:339349.

Chen CC, Chen N, Lau LF. 2001. The angiogenic factors Cyr61 and connective tissue growth factor induce adhesive signaling in primary human skin fibroblasts. J Biol Chem 276:10443-10452.

Chen CC, Lau LF. 2009. Functions and mechanisms of action of CCN matricellular proteins. Int J Biochem Cell Biol 41:771-783.

Chen SJ, Yuan W, Lo S, Trojanowska M, Varga J. 2000. Interaction of smad3 with a proximal smad-binding element of the human alpha2(I) procollagen gene promoter required for transcriptional activation by TGF-beta. J Cell Physiol 183:381-392.

Cristofalo VJ, Lorenzini A, Allen RG, Torres C, Tresini M. 2004. Replicative senescence: A critical review. Mech Ageing Dev 125:827-848.

Dimri GP, Lee X, Basile G, Acosta M, Scott G, Roskelley C, Medrano EE, Linskens M, Rubelj I, Pereira-Smith 0, Peacocke M, Campisi J. 1995. A biomarker that identifies senescent human cells in culture and in aging skin in vivo. Proc Natl Acad Sci USA 92:9363-9367.

Eaglstein WH. 1986. Wound healing and aging. Dermatol Clin 4:481-484.

Fisher GJ, Datta S, Wang Z, Li XY, Quan T, Chung JH, Kang S, Voorhees JJ. 2000. c-Jun-dependent inhibition of cutaneous procollagen transcription following ultraviolet irradiation is reversed by all-trans retinoic acid. J Clin Invest 106:663-670.

Fisher GJ, Henderson PA, Voorhees JJ, Baldassare JJ. 1991. Epidermal growth factor-induced hydrolysis of phosphatidylcholine by phospholipase D and phospholipase C in human dermal fibroblasts. J Cell Physiol 146:309317.

Fisher GJ, Kang S, Varani J, Bata-Csorgo Z, Wan Y, Datta S, Voorhees JJ. 2002. Mechanisms of photoaging and chronological skin aging. Arch Dermatol 138:1462-1470.

Fisher GJ, Quan T, Purohit T, Shao Y, Cho MK, He T, Varani J, Kang S, Voorhees JJ. 2009. Collagen fragmentation promotes oxidative stress and elevates matrix metalloproteinase-1 in fibroblasts in aged human skin. Am J Pathol 174:101-114.

Fisher GJ, Varani J, Voorhees JJ. 2008. Looking older: Fibroblast collapse and therapeutic implications. Arch Dermatol 144:666-672.

Fisher GJ, Voorhees JJ. 1998. Molecular mechanisms of photoaging and its prevention by retinoic acid: Ultraviolet irradiation induces MAP kinase signal transduction cascades that induce AP-1-regulated matrix 
metalloproteinases that degrade human skin in vivo. J Investig Dermatol Symp Proc 3:61-68.

Fisher GJ, Wang ZQ, Datta SC, Varani J, Kang S, Voorhees JJ. 1997. Pathophysiology of premature skin aging induced by ultraviolet light. N Engl J Med 337:1419-1428.

Fligiel SE, Varani J, Datta SC, Kang S, Fisher GJ, Voorhees JJ. 2003. Collagen degradation in aged/photodamaged skin in vivo and after exposure to matrix metalloproteinase-1 in vitro. J Invest Dermatol 120:842-848.

Going JJ, Stuart RC, Downie M, Fletcher-Monaghan AJ, Keith WN. 2002. 'Senescence-associated' beta-galactosidase activity in the upper gastrointestinal tract. J Pathol 196:394-400.

Hall MC, Young DA, Waters JG, Rowan AD, Chantry A, Edwards DR, Clark IM. 2003. The comparative role of activator protein 1 and Smad factors in the regulation of Timp-1 and MMP-1 gene expression by transforming growth factor-beta 1. J Biol Chem 278:10304-10313.

Holt DR, Kirk SJ, Regan MC, Hurson M, Lindblad WJ, Barbul A. 1992. Effect of age on wound healing in healthy human beings. Surgery 112:293-297; discussion 297-298.

Jokinen J, Dadu E, Nykvist P, Kapyla J, White DJ, Ivaska J, Vehvilainen P, Reunanen H, Larjava H, Hakkinen L, Heino J. 2004. Integrin-mediated cell adhesion to type I collagen fibrils. J Biol Chem 279:31956-31963.

Khorramizadeh MR, Tredget EE, Telasky C, Shen Q, Ghahary A. 1999. Aging differentially modulates the expression of collagen and collagenase in dermal fibroblasts. Mol Cell Biochem 194:99-108.

Kireeva ML, Mo FE, Yang GP, Lau LF. 1996. Cyr61, a product of a growth factor-inducible immediate-early gene, promotes cell proliferation, migration, and adhesion. Mol Cell Biol 16:1326-1334.

Klein CE, Dressel D, Steinmayer T, Mauch C, Eckes B, Krieg T, Bankert RB, Weber L. 1991. Integrin alpha 2 beta 1 is upregulated in fibroblasts and highly aggressive melanoma cells in three-dimensional collagen lattices and mediates the reorganization of collagen I fibrils. J Cell Biol 115:1427-1436.

Lau LF, Lam SC. 1999. The CCN family of angiogenic regulators: The integrin connection. Exp Cell Res 248:44-57.

Leask A, Abraham DJ. 2006. All in the CCN family: Essential matricellular signaling modulators emerge from the bunker. J Cell Sci 119:4803-4810.

Matrisian LM. 1994. Matrix metalloproteinase gene expression. Ann NY Acad Sci 732:42-50.

Mo FE, Muntean AG, Chen CC, Stolz DB, Watkins SC, Lau LF. 2002. CYR61 (CCN1) is essential for placental development and vascular integrity. Mol Cell Biol 22:8709-8720.

Perbal B. 2004. CCN proteins: Multifunctional signalling regulators. Lancet 363:62-64.

Quan T, He T, Kang S, Voorhees JJ, Fisher GJ. 2004. Solar ultraviolet irradiation reduces collagen in photoaged human skin by blocking transforming growth factor-beta type II receptor/Smad signaling. Am J Pathol 165:741-751.
Quan T, He T, Shao Y, Lin L, Kang S, Voorhees JJ, Fisher GJ. 2006. Elevated cysteine-rich 61 mediates aberrant collagen homeostasis in chronologically aged and photoaged human skin. Am J Pathol 169:482-490.

Quan T, He T, Voorhees JJ, Fisher GJ. 2001. Ultraviolet irradiation blocks cellular responses to transforming growth factor-beta by down-regulating its type-II receptor and inducing Smad7. J Biol Chem 276:26349-26356.

Quan T, He T, Voorhees JJ, Fisher GJ. 2005. Ultraviolet irradiation induces Smad7 via induction of transcription factor AP-1 in human skin fibroblasts. J Biol Chem 280:8079-8085.

Quan T, Qin Z, Xia W, Shao Y, Voorhees JJ, Fisher GJ. 2009. Matrixdegrading metalloproteinases in photoaging. J Investig Dermatol Symp Proc 14:20-24.

Quan T, Qin Z, Xu Y, He T, Kang S, Voorhees JJ, Fisher GJ. 2010. Ultraviolet irradiation induces CYR61/CCN1, a mediator of collagen homeostasis, through activation of transcription factor AP-1 in human skin fibroblasts. J Invest Dermatol 130:1697-1706.

Quan T, Qin Z, Robichaud P, Voorhees JJ, Fisher GJ. 2011a. CCN1 contributes to skin connective tissue aging by inducing age-associated secretory phenotype in human skin dermal fibroblasts. J Cell Commun Signal 5:201-207.

Quan T, Qin Z, Shao Y, Xu Y, Voorhees JJ, Fisher GJ. 2011b. Retinoids suppress cysteine-rich protein 61 (CCN1), a negative regulator of collagen homeostasis, in skin equivalent cultures and aged human skin in vivo. Exp Dermatol 20:572-576.

Raine-Fenning NJ, Brincat MP, Muscat-Baron Y. 2003. Skin aging and menopause: Implications for treatment. Am J Clin Dermatol 4:371-378.

Tiger CF, Fougerousse F, Grundstrom G, Velling T, Gullberg D. 2001. alpha11beta1 integrin is a receptor for interstitial collagens involved in cell migration and collagen reorganization on mesenchymal nonmuscle cells. Dev Biol 237:116-129.

Varani J, Dame MK, Rittie L, Fligiel SE, Kang S, Fisher GJ, Voorhees JJ. 2006. Decreased collagen production in chronologically aged skin: Roles of age-dependent alteration in fibroblast function and defective mechanical stimulation. Am J Pathol 168:1861-1868.

Varani J, Perone P, Warner RL, Dame MK, Kang S, Fisher GJ, Voorhees JJ. 2008. Vascular tube formation on matrix metalloproteinase-1-damaged collagen. Br J Cancer 98:1646-1652.

Varga J, Rosenbloom J, Jimenez SA. 1987. Transforming growth factor beta (TGF beta) causes a persistent increase in steady-state amounts of type I and type III collagen and fibronectin mRNAs in normal human dermal fibroblasts. Biochem J 247:597-604.

Weinstock MA. 1994. Epidemiology of nonmelanoma skin cancer: Clinical issues, definitions, and classification. J Invest Dermatol 102:4S-5S.

White LA, Mitchell TI, Brinckerhoff CE. 2000. Transforming growth factor beta inhibitory element in the rabbit matrix metalloproteinase-1 (collagenase-1) gene functions as a repressor of constitutive transcription. Biochim Biophys Acta 1490:259-268. 